\title{
Participation in an online community of high school physics teachers
}

\author{
Michael Nadeau, ${ }^{1}$ Bahar Modir, ${ }^{1, *}$ Robynne M. Lock, ${ }^{1}$ and William G. Newton ${ }^{1}$ \\ ${ }^{1}$ Department of Physics \& Astronomy, Texas A\&M University-Commerce, Commerce, TX, 75429, USA
}

We use the theory of community of practice to describe high school physics teachers' participation in the context of an online discussion board as part of a new online Master program in physics with teaching emphasis. In each class, the core component of the design, requires students to participate in weekly problem solving and bi-weekly reading discussion boards. We apply social network analysis measures to quantify the community interactions formed in the context of a nine-week summer course of classical mechanics for educators. Our findings revealed fluctuations in the problem solving discussion boards; a result of the structural features of the course. The participation in the reading discussion boards showed a less variation comparatively. To further analyze the results and reveal the course's structural features, we characterized posts by looking for emergent themes. 


\section{INTRODUCTION}

In recent years, different learning programs have led to the development of various communities of students, faculty, and LAs engaged in authentic physics practices around the shared goal of becoming better learners, teachers, or both [1-3]. Socially contextualized theories of learning, such as Wenger's Community of Practice ( $\mathrm{CoP})$, provide a useful framework for uncovering underlying structures of communities and revealing factors that might affect learning [4]. A CoP has three essential aspects: 'joint enterprise', 'mutual engagement', and a 'shared repertoire'. Learning occurs through community members engaging in regular practices within collaborations. This requires a context with a proper social and technical support structure [5]. Irving and Sayre have identified the pedagogical and structural features in an advanced physics lab that promoted formation and enculturation process of a CoP. For example, long and difficult experiments, encouraged students to reach out to other groups for help, thus promoting the distribution of lab expertise among them [1]. Continued participation can enhance a learner's ways of practice, ranging from being on the periphery of a $\mathrm{CoP}$ to becoming a central member [2]; what Wenger termed a "trajectory" [4]. In this study, we introduce a new community of practice as part of an online Master of Science in Physics with Teaching Emphasis for in-service high school teachers at Texas A\&M University-Commerce. Building on the previous research [1], in our preliminary effort, we investigate what kind of practices emerge from our online community and due to what structural features.

The program has designed a curriculum around 6 new master's online classes intended to reinforce physics teachers' advanced content knowledge and introduce them to the latest results of physics education research. The overarching goal of the program is to prepare physics educators to teach up to the community college level. At the class level, the shared goal is to strengthen teachers' knowledge of the advanced subject content, so they can promote understanding in their own students. A core component of each class is participation in a series of online discussion forums to engage students in authentic practices of learning and teaching physics. Students interact with each other through written posts-in the form of replying to another student's post or initiating a post- to negotiate norms, construct meaning and experiences, and share methods. In other words, developing a repertoire of practices.

Over the span of the semester, the online course we investigated had dynamic underlying structures, such as the variation in the difficulty of content. As a result, we expected that community activity was bound to fluctuate over this time period. We use social network analysis (SNA) measures to gauge community activity and interaction. The SNA tool has been employed more frequently in recent years to better understand the social dimension of learning physics [6-8]. We

*bahar.modir@tamuc.edu use SNA dyadic and reciprocity measures to reveal the overall trend of our community. We then find an emergent coding scheme linking practices to structural features. To uncover the structural features, the substance of member's posts are compared to each other.

\section{CONTEXT}

Approximately 50\% of the program's participants do not hold a bachelor's degree in physics and nearly $50 \%$ are out of state. Currently, the program enrolls 60 students and has nearly 30 students in each course, with two courses being offered each semester. Depending on the semester of entrance to the program, students have the option of taking any of the available core courses, though they are encouraged to begin with the Math Methods course. The graduate-level course in our study, Classical Mechanics for Educators, spanned over nine weeks of a summer semester. During that time, enrolled students participated in two separate online discussion boards. The "problem-solving" discussion board required students to write at least two posts a week regarding their impressions and challenges in applying physics concepts, deploying pedagogical strategies, and employing mathematical techniques. Many of these conversations derived their substance from the content of video-based lectures on math techniques, questions presented in the videos, assignments, and personal experiences. In the "reading" discussion board, students were asked to reflect on assigned reading by authoring 3 posts approximately every other week, as there were only 6 reading assignments - providing them with up-to-date results from PER. In each of these discussion boards, students were allowed to post more than the limit.

\section{METHODS FOR QUANTITATIVE ANALYSIS}

We used SNA to measure and better resolve changes in weekly community engagement, which could provide important context for characterizing student communication and unveiling influential course structures. In SNA, elements and their respective associations can be graphically depicted with "nodes" and "edges". We applied SNA to both the problemsolving and reading discussion boards by representing class members as nodes (circles), and the communication between members, the reply posts, as edges (lines) [9]. An edge forms when a student authors a reply to its intended target. We used measures of dyad connections to quantify our networks. The number of dyad connections tallies how many edges are present in a single network. Since edges are always correlated with associations between pairs of nodes, it provides a useful measure of community activity. For example, a dyad connection measure of 35 would indicate that 35 unique pairs of the community's individuals were engaged in some form of communication during that week. Frequently, pairs of students would write to one another. To numerically gauge interaction within our networks, we turned our attention to bi-directional edges. A bi-directional edge is an indicator that an instance of two-way communication has occurred between a unique pair 


\section{Problem Solving Discussion}
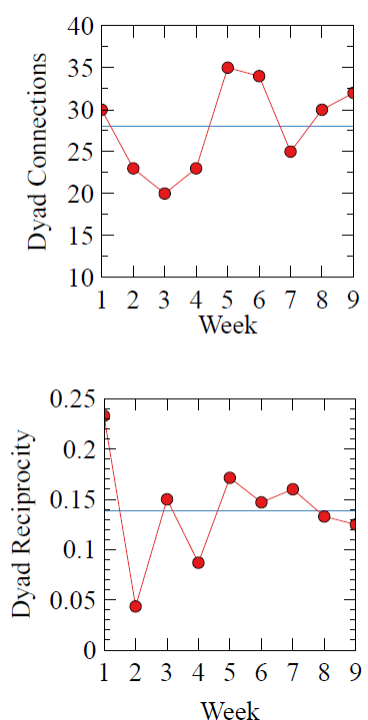

Reading Discussion
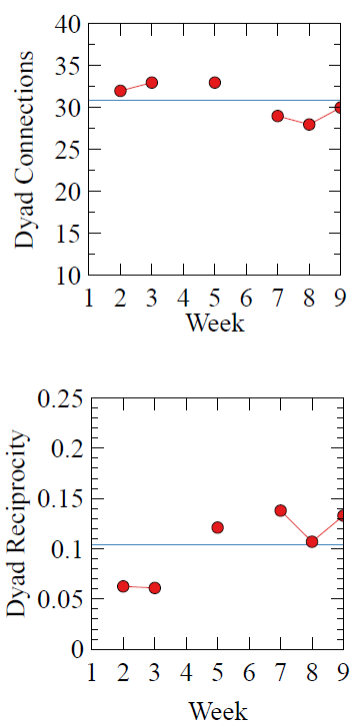

FIG. 1. Graphs showing the weekly dyad connection values and dyad reciprocity for both discussion boards. The horizontal reference lines mark the average values. Gaps in the trend line for the reading discussion show the weeks in which posts were not assigned

of individuals. Therefore, we reason that a greater quantity of these sorts of edges reflects a higher level of community-wide interaction. Dyad reciprocity, which is a convenient measure of this sort of interaction, as shown in Eq. 1, is a determination of the fraction of bi-directional edges.

$$
\text { Reciprocity }=\frac{\text { number of bi-directional edges }}{\text { number of edges }}
$$

We counted the number of edges and bi-directional edges in each week and applied the SNA measures to each discussion board to identify the trends for community activity over the course of the semester. There were 29 students enrolled in the class and 22 participated in this study, which resembles a large section of the community. Replies associated with the study's non-participants were disregarded completely. This removal had little effect on our analysis, as most of the active members participated in the study.

\section{QUANTITATIVE RESULTS}

The graphs in Fig.1, which display the weekly values for dyad connections and dyad reciprocity in each type of discussion board, shows that community participation in the problem-solving discussion board varied throughout the semester, while activity in the reading discussion board was more uniform from week to week with a greater level of activity, as reflected by the overall average. The difference in the minimum number of required posts- 2 for the problemsolving discussion board and 3 for the reading discussion board - explains the higher average number of dyad connections in the reading discussion board. We argue that the narrower scope of the posting parameters for the reading dis-

cussion board contributed to a more consistent level of activity throughout the course's duration. The number of dyad connections in the problem-solving discussion boards starts above average in Week 1, trends downwards, then rises to a peak value in Week 5, which is followed by another dip and rebound in the later weeks of the course. In comparison, the reading discussion boards show considerably more uniformity during the semester. Dyad reciprocity also fluctuates in the problem-solving discussion boards, but does so in a more dramatic, saw-tooth pattern. Some of this dynamicity is likely due to the sensitivity of the calculation: small differences in the number of bi-directional edges can have a significant impact on the calculation's outcome.

The overall average dyad reciprocity is lower than the average found in the problem-solving discussion boards. Some of these observations concerning reciprocity goes beyond the artifacts of a calculation. It hints at the inherent volatility of student interaction, which is somewhat independent of overall activity. Typically, students will engage in back-and-forth communication when they have a need or are responding to a need, such as seeking assistance on a problem, or eliciting advice on teaching strategies.

At a surface level, some of these discrepancies between discussion boards revealed by the analysis can be explained by the different requirements for participation. The dynamic nature of the problem-solving board was a compelling feature that prompted a critical question: which underlying structures could be influencing the disparities observed in the level of community activity?

\section{METHODS FOR QUALITATIVE ANALYSIS}

We conducted a qualitative analysis to thoroughly review individual posts in an attempt to find the underlying structures that influence community engagement. By carefully reviewing the content of posts, coupled with guidance from the dyad connections graphs, it was possible to find particular themes connected to contrasting weeks of the highest and lowest levels of activity. Researchers first coded each week's discussion board individually, followed by detailed discussions of what was seen in the data, reaching $98 \%$ agreement. These two steps resulted in a codebook for the emergent themes. We paid attention to the artifacts such as shared links, keywords expressing a quality (e.g., perceiving a video to be challenging), or words relating the posts to the course structure (e.g., 'the video made me think...'). We applied the codebook against our data, looking for all possible course structures underlying the emerging themes. We observed that different course structures can lead to the same post category, but its effect on the community can vary. For example, some of the posts involving pedagogy were attracting the members' attention more, resulting in follow-up discussions within the same block of posts, as opposed to posts that focused on a particular prompt by the instructor. On the other hand, some of the structures promoted different themes. 
TABLE I. Emergent themes and their corresponding structures

\begin{tabular}{c|l}
\hline \hline Themes & Structures \\
\hline \hline Socialization & 'Introduce yourself' forum, off-topic \\
\hline Triggered metacognition & prompt by instructor, video structure \\
\hline Resource sharing & content difficulty, heavy mathematical emphasis \\
\hline Comfort level with concepts and mathematical techniques & content difficulty, heavy mathematical emphasis, program structure \\
\hline Mapping between math and concepts & content difficulty, heavy mathematical emphasis, video structure \\
\hline \hline Teaching practices & prompt by instructor, off-topic, program structure \\
\hline \hline
\end{tabular}

\section{QUALITATIVE RESULTS}

Within the online class environment the design nature of several factors encouraged students to post on the discussion boards. There were times during the videos in which the instructor presented a question, such as "how do you teach this concept in your class?' Also, mathematical errors in the video or the complexity of the mathematical derivations initiated discussions. Furthermore, students conversed about their comfort level with the video content, as some of the introduced materials contained novel concepts and techniques. The program structure promoted discussions around the role of the course sequence in students' success in the program. During the weeks that homework assignments were due, the number of help-seeking posts increased. A few of the other discussions did not relate to course structures but did fall within the domain of the community by focusing on the issues that high school physics teachers face. These types of conversations are important as they define what the community members care about. Additionally, students supported each other by expressing appreciation for the materials shared or the assistance offered by community members. We call all of the factors that stem from the domain of the community 'off-topic'. Participation in the 'Introduce yourself forum!' helped to define the domain of the community by identifying members' academic background and establishing what members wanted to get out of the program. Two structures promoted discussions in the reading forum: assigned articles and instructional prompts that asked students to relate the reading assignments to their teaching practices.

These structures cultivated discussions that revolved around course content, teaching, metacognition and resource sharing. A thorough survey of the contents of the posts for the problem-solving discussion board revealed a diverse set of common themes, as shown in Table I. In comparison, a survey of the reading discussion board uncovered a small subset of the themes exhibited in the problem-solving forum. The limited number of themes- triggered metacognition and teaching practices- stemming from the instructional prompts, which directed students to make links between reading content and teaching practices, suggests that, when it comes to the assigned readings, online communication is more constrained.

The above-mentioned structures resulted in the following themes, found in the problem-solving discussion board:

Socialization-This theme was characterized by posts that contribute to establishing the CoP by overtly communicating the community's practice and norms. This is apparent in the following excerpt, in which a student is trying to prompt one of their peers to more clearly communicate their thoughts to the community:

S1: I'm interested in what you've posted but not all of us are current teachers. Is CP classical physics? What are CAS and UP?...I'm interested in what you have to say and the perspectives you bring to the class.

Though Socialization was present throughout the semester, it was most prominent in Week 1 . This is largely due to the establishment of a new social structure at the start of the course; a subset of these individuals were entering the program and meeting for the first time. More experienced members sought to make the newcomers feel welcome and supported. A hallmark of socializing behavior is back-and-forth communication, which reciprocity is particularly well suited to measure. Week 1 exhibited the greatest measure of dyad reciprocity. In other weeks, this theme presented itself in the form of support and encouragement. Typically, members of the community were quick to acknowledge another student's help or wellcrafted post by authoring appreciative comments.

Triggered metacognition-Prompts by the instructor, especially when students were asked to contemplate how to integrate specific course content within the classes they teach, led to episodes of self-reflection. Metacognition was also a common refrain when there was an alignment between course materials and the CoP's domain. In other words, course content and resources frequently led to community members reevaluating their beliefs and practices. The excerpt below was situated within a thread that was initiated due to a mistake in a lecture video. The student ponders the value of exposing high school students to mistakes:

S2: Sometimes I wonder if it would be better for me to be able to teach without making any mistakes so that students won't get confused by me doing something wrong, or if it's better to have the mistakes now and then so that they can see an example of how to react when you make a mistake and see that it's okay and that they happen to everyone.

Resource sharing-Frequently, students share software, online resources, or print references in an effort to assist others. This was especially prevalent in weeks with difficult content or a heavy emphasis on mathematics. Interestingly, the presentation of assistance typically precipitated in longer 
posting threads, due to supplemental contributions by other students or expressions of appreciation. In an effort to facilitate community understanding, some members voluntarily created their own materials. This student uses software to visualize a scenario involving a three-dimensional trajectory, described in a video lecture: S3:In case anyone's interested, I produced a parametric plot of position for the first example in the video and attached it to this post.

Comfort level with concepts and mathematical techniques-Weeks with more difficult physics concepts or a heavy emphasis on mathematics typically resulted in more activity. S4: After watching and working through the problems in the week 5 video, I am completely overwhelmed. I got so wrapped up in the math that I kept having to rewind the video...If anyone has tips or tricks for the math in this unit, I'd love to hear them.

Talking about difficulties in a community is an important aspect that reflects established trust $[10,11]$. Conversely, weeks with more approachable content typically lowered activity. This seems to have been one of the driving factors in the problem-solving discussion's noticeable fluctuations in the number of dyad connections.

Mapping between math and concepts-The fact that this is a course on classical mechanics resulted in numerous opportunities for students to carefully consider the connections between mathematics and physical concepts. Weeks with content that contained difficult material, or that had errors were most prone to exhibit posts of this nature. In the example that follows, a student is seeking to explain the correlation between the mathematical elements in the drag formula and the concepts they represent: S1: The squared (or quadratic) term includes the extra $v$ for fast speeds when you're imparting more momentum to the fluid the faster you go. At slow speed, it's just the one $v$ term for the amount of fluid you're hitting in proportion to the speed...

Connecting material to past or future academic experiences-Frequently, students would reflect on how the course's curriculum fits into the greater context of their academic careers. This theme often was a way for students to communicate how previous classes (Math Methods) in the program prepared them for classical mechanics.

S5: Math Methods was a huge help to this unit. By total chance, the notebook I am taking notes in for this unit is the same as the one that I did for Math Methods, so my curl and divergence notes were already out while I was listening to the video. That was a huge help.

In certain cases, a post would relate a narrative of the tribulations or successes in a past undergraduate course. Other posts of this theme, in anticipation of future courses in the program, would make predictions on how the material being learned would have an impact on outcomes in future classes, as influenced by the program's coursework requirement: S6: I am excited to take quantum when the class next gets offered so I can use these skills at that level.

Teaching practices-Given that the domain of this commu- nity largely revolves around high school physics pedagogy, community engagement was enhanced by students initiating conversations on teaching practices. This theme was characterized by class members commenting on a teaching method that they have employed or will employ, or an attempt to apply the course's conceptual or mathematical content in their own classes.

Sometimes this theme would dominate in a particular week, especially if the instructor tried to kick-start the conversation by using a prompt in the video. In Week 4, students were asked to share their approaches for teaching conservative and nonconservative forces. As a result, a number of the posts for the week resembled each other, narrowing the conversation, which is likely a contributing factor for that week's reduction in activity, as reflected in the dyad connections.

In other weeks, broader discussions would develop from student-initiated threads around this theme. Sometimes these sorts of threads would only tangentially relate to the content of the week. However, since these still tended to be within the scope of the community's domain, it easily captured the attention of the class:

S7: This is off topic, but do any of you use the flipped classroom model to teach physics?...Pros? Cons? Your experiences? Thanks!

This particular post in week 5 garnered the wider attention of the community and played a role in enhancing that week's level of community engagement, as reflected in the number of dyad connections. These types of posts provided an opportunity for the members to distribute their expertise [1].

\section{DISCUSSION AND CONCLUSION}

Our data show that structures within a course can affect community activity. The two separate discussion boards built into the course provided an opportunity to conduct a comparative analysis anchored in SNA. Changes in weekly activity and interaction were more noticeable in the problem-solving discussion board. Designing an online learning community of practice can be challenging due to its asynchronous nature impeding real time feedback. The identified structural features in our online learning community might provide insight into what aspects need to be considered in designing an active online community that allows for various needs to be met-the emergent theme of metacognition shows that teachers can combine different goals of learning content and pedagogy to build new goals - this expands the community's domain, which in turn furthers participation. Embedded materials are important aspects that enhance a practitioner's skills if they are challenging enough to promote collaboration. Despite the asynchronous mode of content delivery, the prompts presented in the video encouraged community feedback. In an effort to better characterize the development of our online communities, future investigations will entail a more robust implementation of SNA to the community networks to study its volatile nature. 
[1] P.W. Irving and E.C. Sayre, "Conditions for building a community of practice in an advanced physics laboratory," Phys. Rev. ST Phys. Educ. Res., 10, 1 (2014).

[2] P. Irving and M. Caballero, "Understanding the PICUP community of practice," presented at the Physics Education Research Conference 2017, Cincinnati, OH, 2017, https://www.compadre.org/Repository/document/ServeFile. $\mathrm{cfm} ? \mathrm{ID}=14602 \& \mathrm{DocID}=4779$.

[3] E. W. Close, J. Conn, and H. G. Close. Becoming physics people: Development of integrated physics identity through the Learning Assistant experience. Phys. Rev. ST Phys. Educ. Res., 12, 1 (2016).

[4] E. Wenger, Communities of Practice: Learning, meaning and identity (Cambridge University Press, New York, 1998).

[5] S. Barab, J. Makinster, J. Moore, and D. Cunningham, "Designing and Building an On-Line Community: The Struggle to Support Sociability in the Inquiry Learning Forum," Educational Technology Research and Development, 49, 4 (2001).

[6] J. Bruun and E. Brewe, "Talking and learning physics: Predicting future grades from network measures and Force Concept Inventory pretest scores," Phys. Rev. ST Phys. Educ. Res., 9, 2
(2013).

[7] C.A. Hass, F. Genz, M.B. Kustusch, P.A. Ouimet, K.E. Pomian, E.C. Sayre, and J.P. Zwolak, "Studying Community Development: Network Analytical Approach," in Physics Education Research Conference Proceedings, 2018.

[8] R. Dou and J.P. Zwolak, "Practitioner's guide to social network analysis: Examining physics anxiety in an active-learning setting," Phys. Rev. Phys. Educ. Res., 15, 2 (2019).

[9] E. Brewe, "The Roles of Engagement: Network Analysis in Physics Education Research," Getting Started in PER, edited by C. Henderson and K.A. Harper (American Association of Physics Teachers, College Park, MD, 2018), Reviews in PER Vol. 2, (2018),http://www.per-central.org/items/detail. cfm?ID=14725 Retrieved 3/8/2020.

[10] M. Dancy, A. C. Lau, A. Rundquist, and C. Henderson, "Faculty online learning communities: A model for sustained teaching transformation." Phys. Rev. Phys. Educ. Res., 15, 2 (2019).

[11] L. Dube, A. Bourhis, and R. Jacob. "Towards a typology of virtual communities of practice. Interdisciplinary Journal of Information, Knowledge and Management 1 (2006), p.69-94. 